
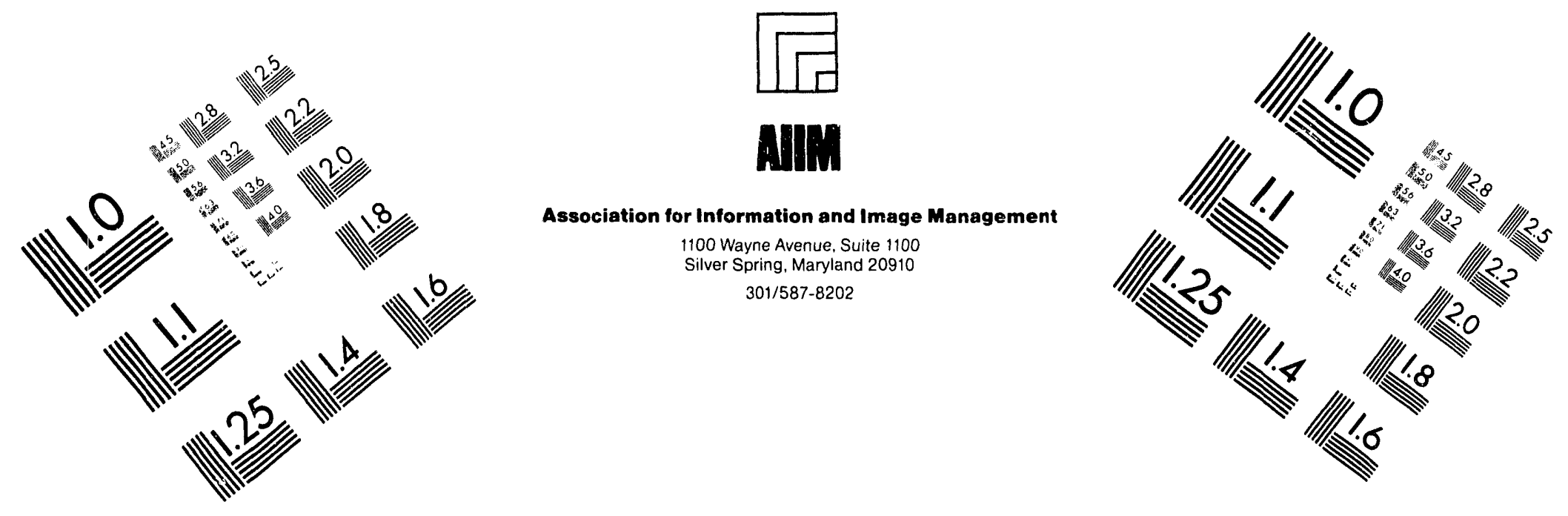

\title{
Centimeter
}

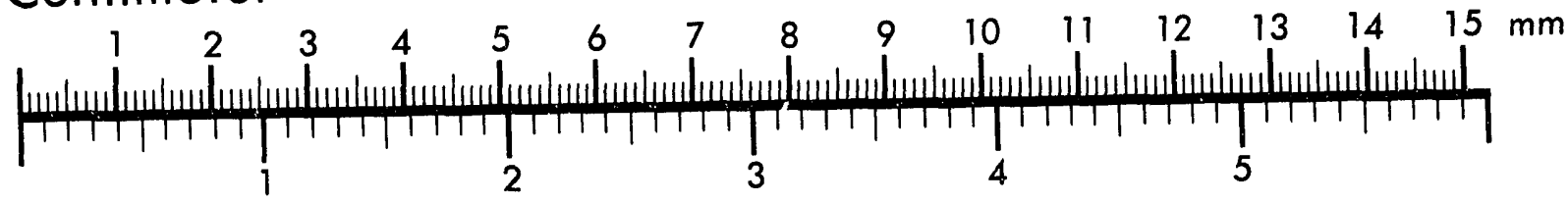
Inches
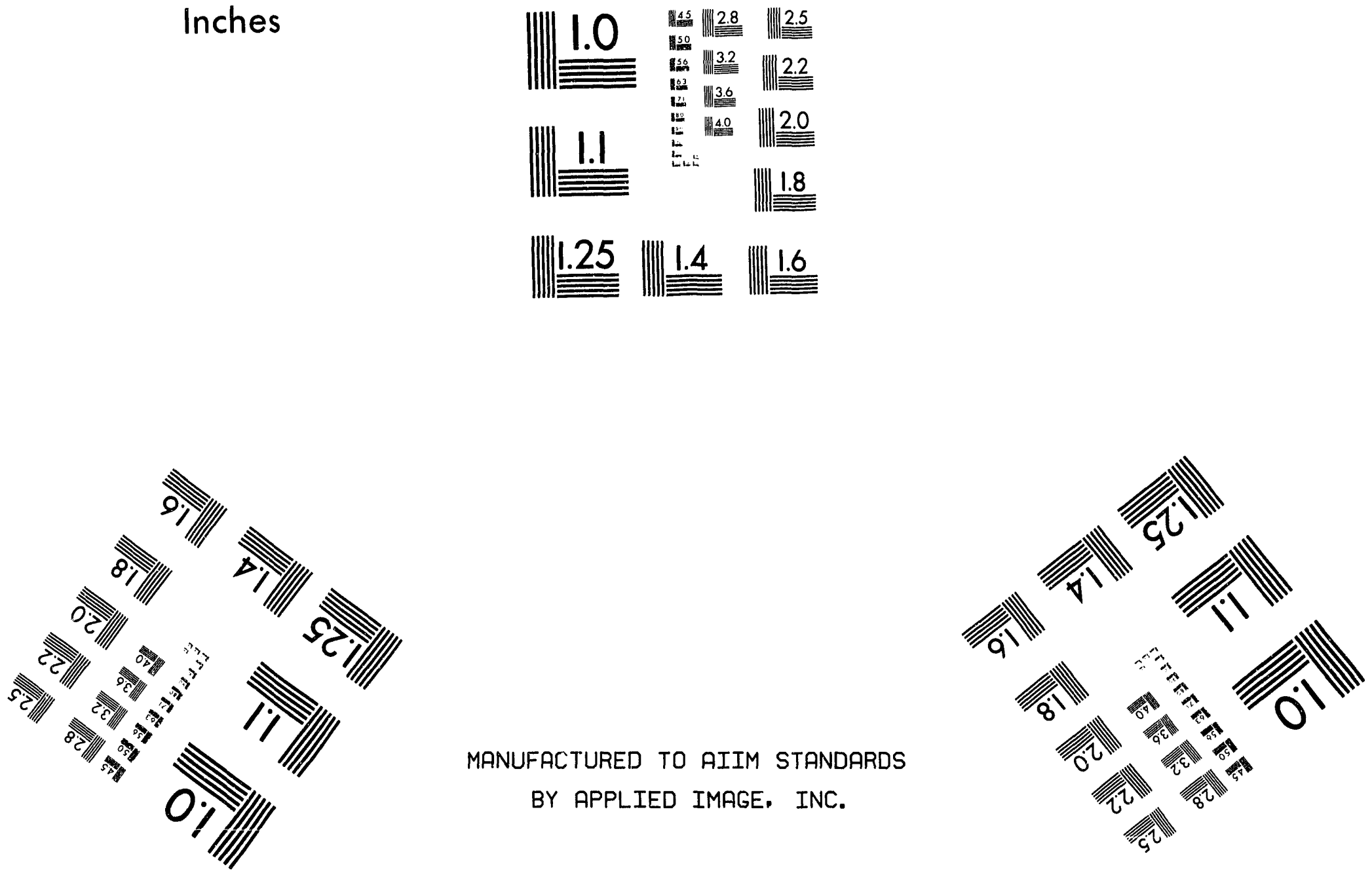

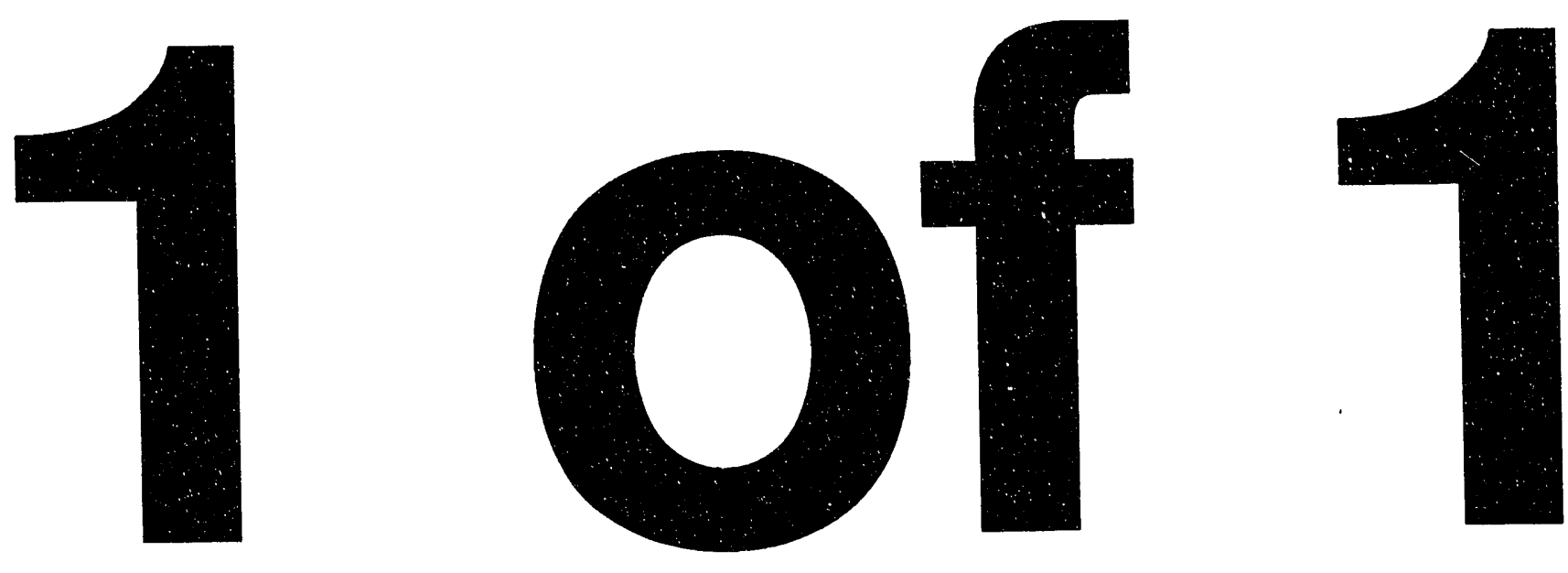
1993 Particle Accelerator Conf., Washington, D.C.

\section{A New Formulation of Longitudinal Coherent Instatilities}

\section{S.Y. Zhang and W.T. Weng}

AGS Department, Brookhaven National Laboratory

Upton, New York 11973

\begin{abstract}
The quadrature response of longitudinal impedance is shuwn to be the effective impedance for the beam instability. The results of the application of this formulation are compared with that obtained using the Robinson-Pedersen approach and the Sacherer integral equation. The formulation is further generalized to the rigid bunch motion using signal analysis method, where a form factor shows up naturally. Finally, the formulation is applied to solve the coupled bunch instabilities. Examples of the AGS Booster and the AGS coupled bunch instabilities are used to illustrate the applications of the formulation.
\end{abstract}

\section{The New Formulation}

In the longitudinal motion, a synchrotron oscillation is modulated by the RF carrier. The beam current induced voltage through the longitudinal impedance may affect the synchrotron oscillation and cause the beam instability.

\section{A. Beam Dynamic Model}

A beam dynamic model is shown in Fig.1, where $s$ is the Laplace operator, $\omega_{O}$ and $\omega_{R F}$ are the revolution and RF frequencies, respectively. $\Delta V_{B}$ is the equivalent $R F$ gap voltage deviation caused by the beam motion, and $\triangle V_{C A V}$ caused by the cavity voltage variation. $I_{B}$ is the beam current amplitude of the fundamental frequency, i.e., the RF frequency. Finally, $Z_{M}(s)$ represents the effective longitudinal impedance with respect to the beam instability. In the block diagram, the upper loop represents the synchrotron oscillation, as shown in $[1]$. The lower loop represents the effects of the beam current to the cavity voltage through the longitudinal impedance.

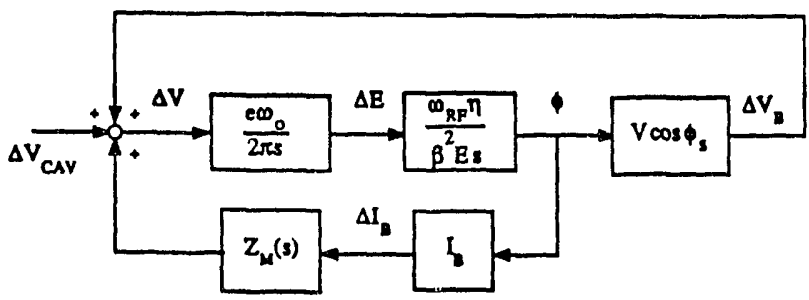

\section{$B$. Impedance}

Fig.1. Beam Dynamic Model

We use both Laplace and Fourier transforms. For instance, an impedance in the Laplace form can be $Z\left(s+j \omega_{R F}\right)$, and its counterpart in the Fourier form is written as $Z\left(\omega+\omega_{R F}\right)$. Consider a general situation of the modulated input and output. Let the input signal of a system be $f(t)$ and the output be $g(t)$. The input signal is assumed to be a low frequency signal $f_{L}(t)$ modulated by an RF carrier, say $\cos \omega_{R F} t$, i.e., we can write, $f(t)=f_{L}(t) \cos \omega_{R F} t$. Using $f(t) \rightarrow F(\omega)$ and $f_{L}(t) \rightarrow F_{L}(\omega)$ to denole the Fourier

* Work performed under the auspices of the U.S. Department of Energy pairs, we have,

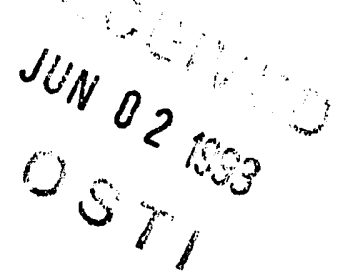

$$
F(\omega)=\frac{1}{2}\left(F_{L}\left(\omega+\omega_{R F}\right)+F_{L}\left(\omega-\omega_{R F}\right)\right)
$$

Also for $f_{1}(t)=f_{L}(t) \sin \omega_{R F} t$, we have,

$$
F_{1}(\omega)=\frac{1}{2 j}\left(F_{L}\left(\omega+\omega_{R F}\right)-F_{L}\left(\omega-\omega_{R F}\right)\right)
$$

Under the modulation of the frequency $\omega_{R F}$, the inphase and quadrature responses of the impedance $Z(\omega)$ are determined by [2],

$$
\begin{aligned}
& Z_{P}(\omega)=\frac{1}{2}\left(Z\left(\omega+\omega_{R F}\right)+Z\left(\omega-\omega_{R F}\right)\right), \\
& Z_{Q}(\omega)=\frac{1}{2 j}\left(Z\left(\omega+\omega_{R F}\right)-Z\left(\omega-\omega_{R F}\right)\right)
\end{aligned}
$$

respectively. We also define $G_{P}(\omega)=F_{L}(\omega) Z_{P}(\omega)$ and $G_{Q}(\omega)=F_{L}(\omega) Z_{Q}(\omega)$. Using $g_{P}(t) \stackrel{L}{\rightarrow} G_{P}(\omega)$ and $g_{Q}(t) \rightarrow G_{Q}(\omega)$ for Fourier pairs, the total response of the modulated signal $F(\omega)$ in (1) through the impedance $Z(\omega)$ is,

$$
g(t)=g_{P}(t) \cos \omega_{R F} t+g_{Q}(t) \sin \omega_{R F} t
$$

Using (1-4), the equation (5) can be shown to be,

$$
G(\omega)=F(\omega) Z(\omega))
$$

therefore (5) is proved. When the beam passes the cavity gap, the in-phase response due to the cavity impedance, which is modulated by $\cos \omega_{R F} t$, provides an almost constant force in the beam synchrotron oscillation, which has little effect on the beam instability. On the other hand, since the quadrature response is modulated by $\sin \omega_{R F} t$ it is in the same fashion as that of the RF driving wave and functions as the same as the RF driving wave. Therefore, if the instability of synchrotron oscillation is concerned, the quadrature response represented by (4) becomes a dominant effect. Thus the effective longitudinal impedance is,

$$
Z_{M}(s)=Z_{Q}(s)=\frac{1}{2 j}\left(Z\left(s+j \omega_{R F}\right)-Z\left(s-j \omega_{R F}\right)\right)
$$

Consider an $\mathrm{RF}$ cavity with the resonant frequency $\omega_{R}$, the shunt resistance $R$, the halfbandwidth $\sigma$, and the quality factor $Q$. Under the conditions of $Q \gg 1, \omega_{R F} \approx \omega_{R} \gg \omega \approx \omega_{s}$, and also $\left.\left|\omega_{R}^{2}-\omega_{R F}^{2}\right| \gg\right|^{2} \mid=\omega^{2}$, the longitudinal impedance of the $\mathrm{RF}$ cavity is,

$$
Z_{M}(s)=\frac{-R \sigma^{2} \tan \phi_{Z}}{s^{2}+2 \sigma s+\sigma^{2}\left(1+\tan ^{2} \phi_{Z}\right)}
$$

where the detuning angle $\phi_{Z}=\tan ^{-1}\left(\left(\omega_{R F}-\omega_{R}\right) / \sigma\right)$. C. Stability

To study the beam stability under the influence of the longitudinal impedance of $R F$ cavity, we can write the following equation from Fig.1,

$$
\phi=\frac{\mathrm{e} \omega_{O} \omega_{R F} \eta V \cos \phi_{S}}{2 \pi \beta^{2} E s^{2}} \phi+\frac{\mathrm{e} \omega_{O} \omega_{R F} \eta}{2 \pi \beta^{2} E s^{2}} Z_{M}(s) I_{B} \phi
$$


Using $\omega_{S}^{2}=-\mathrm{e} \omega_{O} \omega_{R F} \eta V \cos \phi_{S} /\left(2 \pi \beta^{2} E\right)$ and the beam and generator current ratio $Y=I_{B} / I_{G O}=I_{B} R / V$, the characteristic equation of the system becomes,

$$
s^{2}+\omega_{S}^{2}=\frac{\omega_{S}^{2} Y \sigma^{2} \tan \phi_{Z} / \cos \phi_{S}}{s^{2}+2 \sigma s+\sigma^{2}\left(1+\tan ^{2} \phi_{Z}\right)}
$$

Using Routh-Hurwitz table, it is straightforward to find the stability conditions as $\tan \phi_{Z}>0$ and $Y \tan \phi_{Z} \cos ^{2} \phi_{Z}<\cos \phi_{S}$, which are called the first and second Robinson criteria, respectively.

In deriving the transfer function $Z_{M}(s)$ in Fig.1, the in-phase and quadrature transfer functions $Z_{P}(s)$ and $Z_{Q}(s)$ are used. In the Robinson - Pedersen approach [3], the beam to cavity phase and amplitude transmissions and their cross transmissions $Z_{p p}(s)$, $Z_{a a}(s)$, and $Z_{p a}(s), Z_{a p}(s)$ are used to derive the total equivalent transmission from the beam phase variation to the induced cavity voltage phase deviation. In Fig.2, typical step responses of the transfer functions are plotted, which show the difference between the two types of the transfer functions, and the two approaches as well.

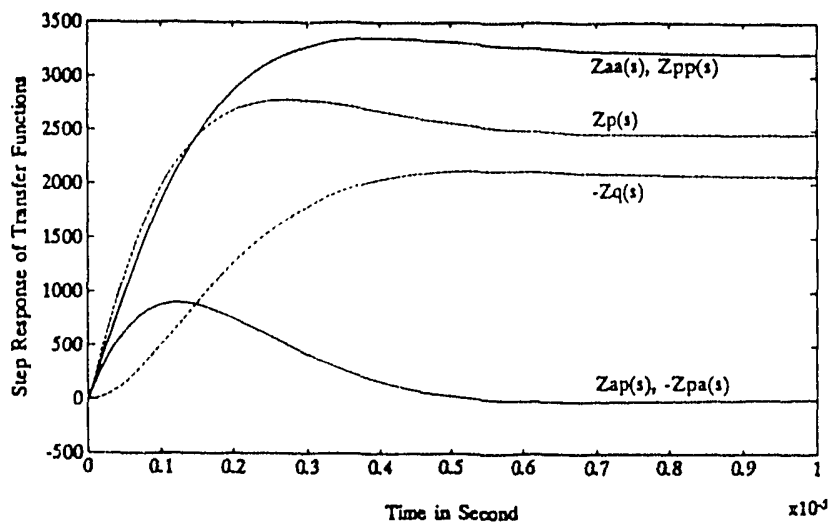

Fig.2. Step Responses for the Transfer Functions.

Using Sacherer integral equation [4] for the dipole motion, we get the following equation,

$$
s^{2}+\omega_{S}^{2}=\frac{8 \pi \omega_{S}^{2} I_{0}}{V \cos \phi_{S}}\left(\int_{0}^{\infty} J_{1}^{2}(r) \frac{d \psi_{0}}{d r} d r\right) Z_{M}(s)
$$

where $I_{0}$ is the beam average current, $\psi_{0}$ is the bunch stationary distribution, and $r$ is the amplitude of the beam phase ascillation. Substituting (8), the equation (11) is shown to be equivalent to (10), except for a scaling difference.

\section{Generalize the Formulation}

To generalize the formulation to the rigid bunch motion, the beam current signal needs to be analyzed. For each frequency component in the signal, the corresponding effective impedance can be found, which needs only a trivial modification from the results shown in Section I. The summation of the effects of the impedance due to each component in the signal is the force the beam received.

\section{A. Signal of rigid bunch motion}

Let $T_{R F}$ be the RF period, $T_{R F}=2 \pi / \omega_{R F}$, a beam longitudinal current signal with $N$ particles in a bunch is,

$$
i(t)=N \mathrm{e} \sum_{k=-\infty}^{\infty} \delta\left(t-k T_{R F}+\tau \cos \omega_{S} k T_{R F}\right)
$$

where $\tau$ is the synchrotron oscillation amplitude in time. The spectrum of this signal is calculated as I 1 .

$$
I(\omega)=2 \pi I_{0} \sum_{p, m=-\infty}^{\infty} j^{m} J_{m}(-\tau) \delta\left(\omega-p \omega_{R F}-m \omega_{S}\right)(1: 3)
$$

We further assume that the bunches have a Gaussian distribution, which is thosen for convenience, and with an effective bunch length ${ }^{\prime} L$. Using the phase oscillation amplitude $r=\omega_{R F} \tau$ the equation (13) becomes $(1)$,

$$
\begin{gathered}
I(\omega)=2 \pi I_{0} \sum_{p, m=-\infty}^{\infty} j^{m} J_{m}\left(r \omega / \omega_{R F}\right) \\
\times e^{-\left(r_{L} \omega / \omega_{R F}\right)^{2} / 32} \delta\left(\omega-p \omega_{R F}-m \omega_{S}\right)
\end{gathered}
$$

\section{B. Generalization}

To compare the rigid bunch signal represented by (14) with the idealized signal, we may write the signal used to develop the formulation in Section I as $i_{1}(t)=I_{B} r \cos \omega_{S} t \sin \omega_{R F} t$, whose spectrum is,

$$
I_{1}(\omega)=2 \pi I_{B} \frac{r}{4 j} \sum_{p, m= \pm 1}(-p) \delta\left(\omega-p \omega_{R F}-m \omega_{S}\right)
$$

The first difference between the real rigid bunch motion signal represented by (14) and the idealized signal (15) is that (14) contains not only RF frequency modulation but also RF harmonics modulation, i.e., by the frequencies $p \omega_{R F},|p|>1$. It is shown $[1 \mid$ that $Z_{M}(\omega)=Z_{Q}(\omega)$ is valid not only for the RF frequency modulation, but also for the RF harmonic modulation. For the carrier with the frequency $p \omega_{R F}$, the variable $\omega_{R F}$ in (4) should however be replaced by $p \omega_{R F}$. In the system synthesis, firstly these frequency components in the rigid bunch motion signal should be identified, then the corresponding longitudinal impedances should be used to find the induced forces. The combined force is the one the beam received.

The second difference of (14) from (15) is that it contains not only dipole motion but also high mode motion, i.e., $m \omega_{s},|m|>1$.

The third difference is that in (14), the spectrum amplitude is affected by che Bessel function, the bunch distribution and the bunch length. The combined influence of these factors can be called a form factor.

\section{Form factor}

Consider the most important case of dipole motion with RF frequency modulation, where $p= \pm 1$ and $m= \pm 1$. We write (14) as,

$$
\begin{gathered}
I(\omega)=8 \pi I_{0} J_{1}\left(r|\omega| / \omega_{R F}\right) e^{-\left(r_{L} \omega / \omega_{R F}\right)^{2} / 32} \\
\times \frac{1}{4 j} \sum_{p, m= \pm 1}(-p) \delta\left(\omega-p \omega_{R F}-m \omega_{S}\right)
\end{gathered}
$$

For the delta distribution, $I_{B}=2 I_{0}$, the form factor can be written as,

$$
\begin{aligned}
F=\frac{I(\omega)}{I_{1}(\omega)} & =\frac{2 J_{1}\left(r|\omega| / \omega_{R F}\right)}{r} e^{-\left(r_{L} \omega / \omega_{R F}\right)^{2} / 32} \\
& \approx \frac{2 J_{1}(r)}{r} e^{-\left(r_{L} \omega / \omega_{R F}\right)^{2} / 32}
\end{aligned}
$$

where in the simplification we used $|\omega| \approx \omega_{R F}$.

Consider the longitudinal dipole motion discussed in Section I again. The form factor $F$ in (17) has to be multiplied to the scaling $I_{B}$ in Fig.1, and therefore also to $Y=I_{B} R / V$ in $(10)$. Thus, $Y_{1}=F Y$ will replace $Y$ in the second stability equation. Since $F<1$, the stability margin is extended. 
III. Coupled Bunch Instabilities

A. Coupled bunch motion

Let there be $h$ bunches, and let $n$ be the coupled bunch mode number. There will be $n=0,1, \ldots, h-1$ coupled bunch modes [4]. The spectrum of the signal observed from the wall monitor becomes,

$$
\begin{gathered}
I(\omega)=2 \pi I_{0} \sum_{p, m=-\infty}^{\infty} j^{m} J_{m}\left(r \omega / \omega_{R F}\right) \\
\times e^{-\left(r_{L} \omega / \omega_{R F}\right)^{2 / 32} \delta\left(\omega-p \omega_{R F}-n \omega_{0}-m \omega_{S}\right)}
\end{gathered}
$$

The coupled bunch mode of $n \omega_{0}$ can be assumed to be a rigid wave. For an individual bunch, the modulation effect of the beam current signal due to the coupled bunch mode is demodulated. By the same argument as in Section I, the quadrature response represents the effective longitudinal impedance. Therefore we have the following longitudinal impedance,

$$
Z_{M}(\omega)=\frac{1}{2 j}\left(Z\left(\omega+n \omega_{0}+\omega_{R F}\right)-Z\left(\omega+n \omega_{0}-\omega_{R F}\right)\right)
$$

\section{$B$. Coupled bunch instabilities}

If $n \neq 0$, then the two spectrum lines of the same frequency modulation may be far apart, therefore in general the treatment for the resonator type impedance such as that in Section I cannot be applied, and the spectrum lines may have to be treated separately. Consider the dipole upper sideband at $Z\left(\omega+\omega_{0}+\omega_{R F}\right)$ in (19), and let the real part of the impedance be $R_{1}$. Using $s=j \omega \approx j \omega_{S}$, the stability equation can be written as,

$$
s^{2}+\omega_{S}^{2}=\frac{\omega_{S}^{2} I_{B} F}{2 j V \cos \phi_{S}} Z\left(\omega+\omega_{0}+\omega_{R F}\right) \approx \frac{-\omega_{S} I_{B} F R_{1}}{2 V \cos \phi_{S}} s
$$

Below transition $\cos \phi_{S}>0$, therefore the upper sideband is stable because that the coefficient of $s$ is negative. It follows that the lower sideband at $Z\left(\omega+\omega_{0}-\omega_{R F}\right)$, which has a negative sign in (19), is unstable, and the opposite above transition.

It is interesting to revisit the form factor derived in Section II. We rewrite it as,

$$
F=\frac{2 J_{1}\left(r|\omega| / \omega_{R F}\right)}{r} e^{-\left(r_{L} \omega / \omega_{R F}\right)^{2} / 32}
$$

The simplification of the form factor in (17) cannot be made in the case of the coupled bunch mode, since now $|\omega|$ is not close to $\omega_{R F}$ if $n \neq 0$, and both variables have to be considered in the Bessel function. For high frequency, the influence of $r$ shows up, which is shown in Fig.3.

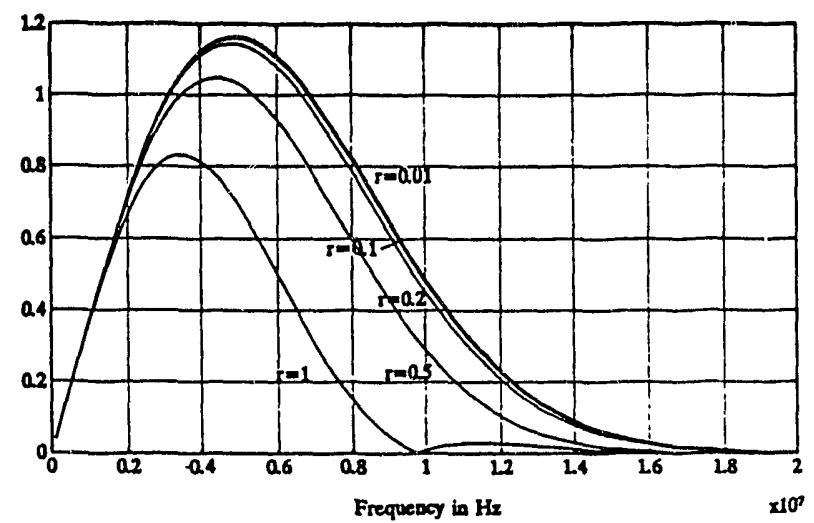

Fig.s. Form Factors with Different r.
In a small range of $r$, for instance between 0.01 to 0.2 in Fig.3, the form factors are approximately the same. This shows the reason why we can assume that the coupled bunch mode is a rigid wave in the instability study.

\section{Examples of the AGS Booster and the AGS}

A coupled bunch instability has been excited in the AGS booster by tuning a test RF cavity [5]. In the booster $h$ is 3 , and $f_{R F}$ was $2.55 \mathrm{MHz}$. In the test, the coupled bunch instability of a dipole mode was observed at the first revolution line, i.e., at $850 \mathrm{KHz}$. which implies that $n=2$. We have $I_{0}=0.082 \mathrm{~A}$. $\phi_{S}=0$, and $V=30 \mathrm{KV}$, and $\tau_{L}=130 \mathrm{nS}$. The RF cavity used to excite the coupled bunch motion has approximately a quality factor 2.5 and a shunt resistance $3 \mathrm{~K} \Omega$, it was tuned at the revolution frequency in the test. To estimate the effective resistance $F R_{1}$, the form factor in (21) is used, where the Gaussian distribution is still used allowing minor errors. The effective resistance of the test cavity is found to be 1 $\mathrm{K} \Omega$ at $850 \mathrm{KHz}$. The growth rate calculated using (20) is $27.7 \mathrm{mS}$, which is close to the test result of $30 \mathrm{mS}$.

In an AGS operation, a coupled bunch instability was observed at the $1.77 \mathrm{GeV}$ front porch, with $f_{R F}=4.18 \mathrm{MHz}$, and $n=11(h=12)$. To find the location of frequency of the exciting resonator, two tests were performed, with $\tau_{L}$ are $46 \mathrm{nS}$ and $70 \mathrm{nS}, I_{0}$ are $0.089 \mathrm{~A}$ and $0.457 \mathrm{~A}, V$ are $260 \mathrm{KV}$ and $184 \mathrm{KV}$, and $f_{S}$ are $1.64 \mathrm{KHz}$ and $1.38 \mathrm{KHz}$, respectively. The observed growth rates are $48 \mathrm{mS}$ and $24 \mathrm{mS}$, respectively. Using a moderate $r=0.1 r_{L}$, the resistance required to generate the observed growth rates are plotted in Fig.4, which shows that at $17.6 \mathrm{MHz}$ the required resistances are crossed. The closest unstable coupled bunch mode frequency is at $17.1 \mathrm{MHz}$, therefore Fig.4 shows a possible location of the exciting resonator. This result agrees to the one obtained by different approaches [6].

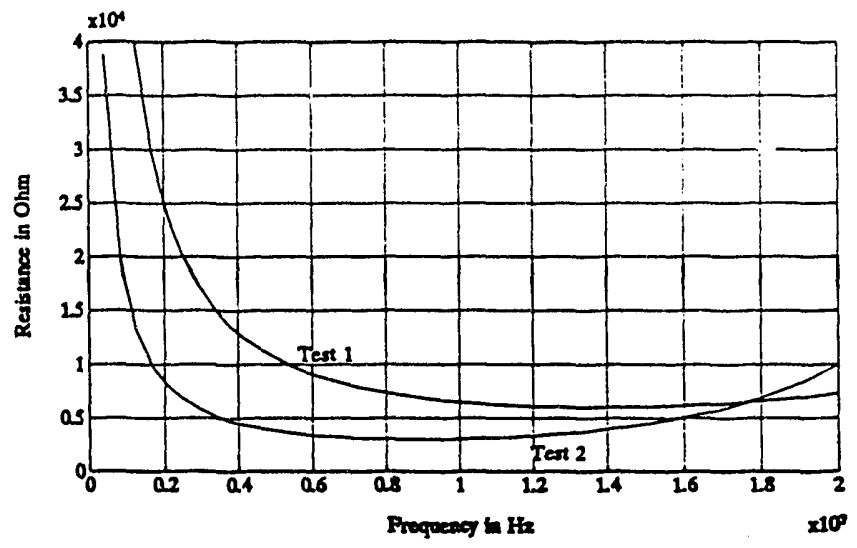

Fig.4. Resistances to Excite the Growth Rates.

\section{Acknowledgment}

The authors would like to thank A. Chao, E.C. Raka, and A.M. Sessler for helpful discussions.

\section{References}

[1] S.Y. Zhang and W.T. Weng, BNL Informal Report, No.48905, May, 1993.

[2] A. Paupolis, 'The Fourier Integral and Its Applications,' New York, McGraw-Hill, 1962.

(3) F. Pedersen, IEEE Trans. NS-22, 1906, 1975.

[4] F. Sacherer, IEEE Trans. NS-24, 1393, 1977.

[5] M. Blaskiewicz, Private communication.

[6] E.C. Raka, Private communication. 

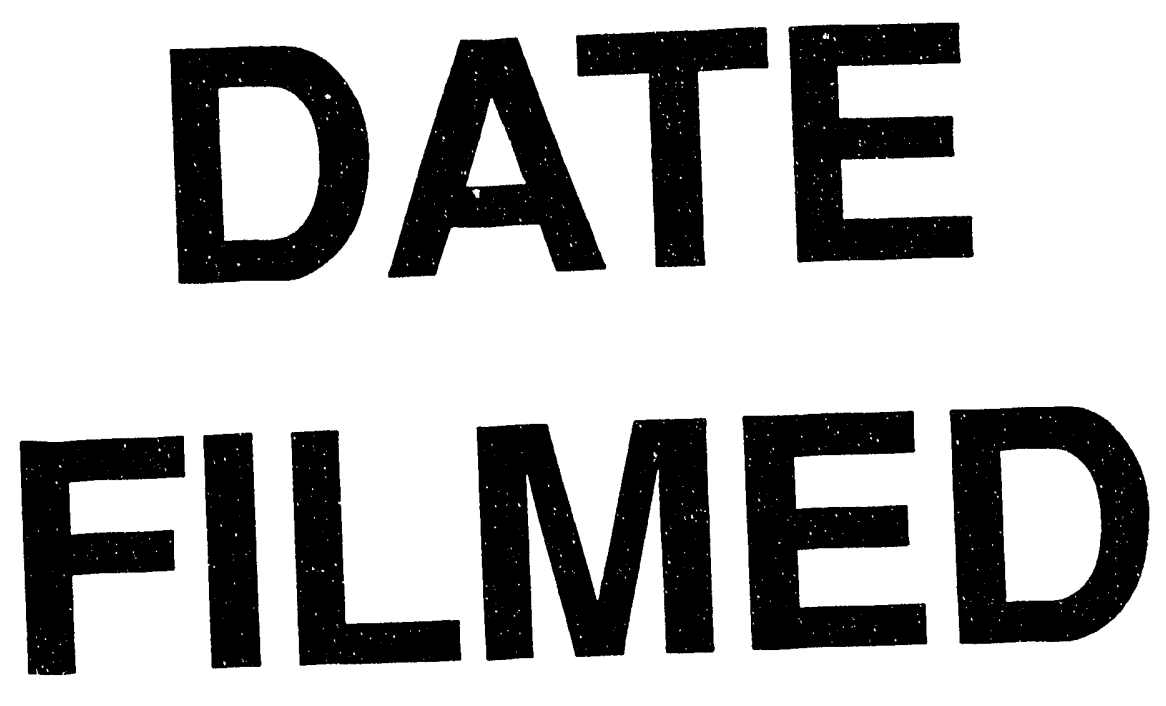

$8 / 4 / 93$
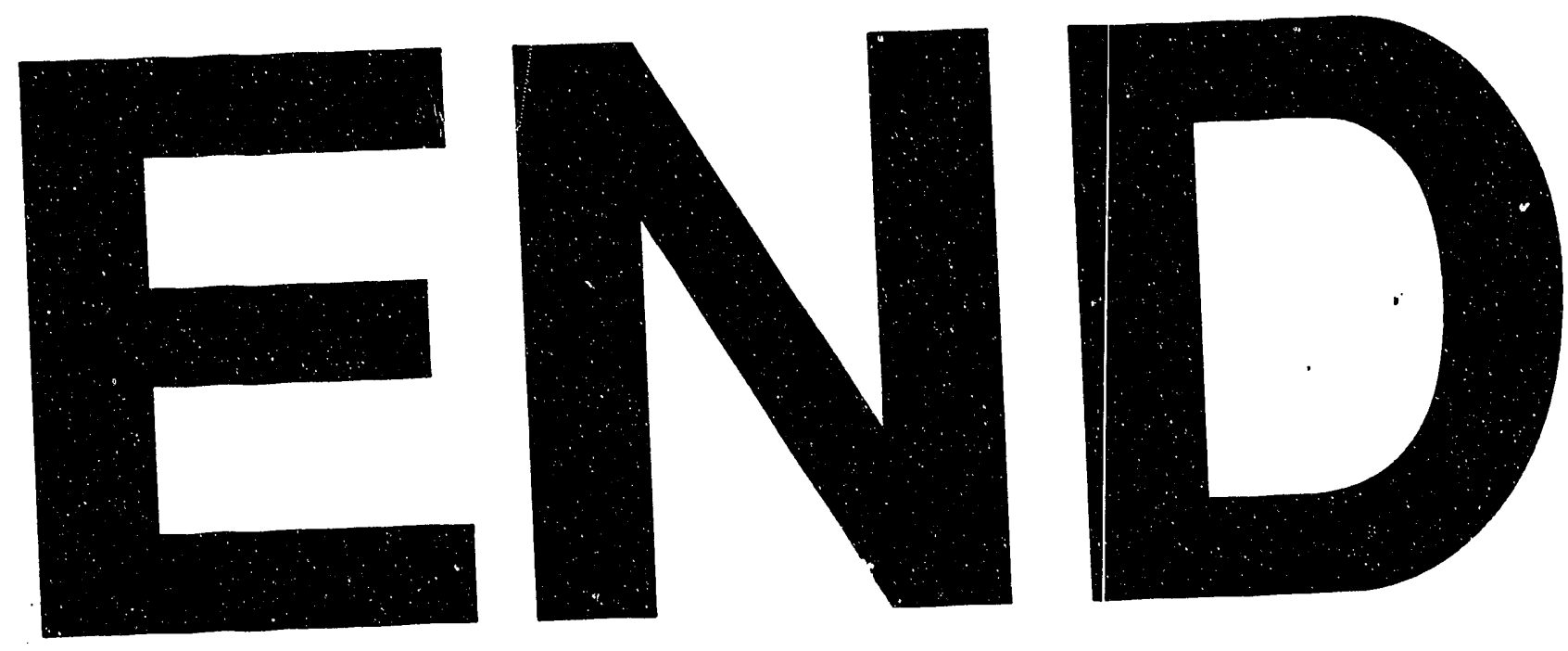
